$$
\frac{1}{B\left(\frac{1}{2}, \frac{\nu}{2}\right)} \int_{x_{\alpha}}^{1} p^{-t}(1-p)^{(\nu-2) / 2} d p=\alpha .
$$

Using the approximation of ordinate over abscissa for the cumulative normal for extreme abscissa we find that $z$ is the abscissa of a cumulative normal which is approximately equal to the power of the $t$-test for alternative $\delta$. In a similar manner the normal approximation to the binomial yields $z=\delta \sqrt{r+1}$ for the sign test. A fixed value of $N$ and $\alpha$ determines $r, \alpha, x_{\alpha}$ and we may solve for $\nu$.

\title{
REFERENCES
}

[1] National Bureau of Standards, Tables of the Binomial Probability Distribution, U. S. Government Printing Office, Washington, D. C.

[2] K. Pearson, Tables of the Incomplete Beta Function, Cambridge University Press, 1934.

[3] Burton H. Camp, "Approximation to the point binomial," Ann. Math. Stat., Vol. 22 (1951), pp. 130-131.

[4] W. G. CocHran, "The efficiencies of the binomial test of significance of a mean and of a correlation coefficient," J. Roy. Stat. Soc., Vol. 100 (1937), pp. 69-73.

[5] W. J. Dixon ANd A. M. Mood, "The statistical sign test," J. Amer. Stat. Assn., Vol. 41 (1946), pp. 557-566.

[6] Murray F. FreEman AND John W. Tukey, "Trangformations related to the angular and the square root," Ann. Math. Stat., Vol. 21 (1950), pp. 607-611.

[7] T. A. Jeeves AND Robert Richards, "A note on the power of the sign test," Ann. Math. Stat., Vol. 21 (1950), p. 618.

[8] W. L. Nicholson, "A computing formula for the power of the analysis of variance test," submitted to Ann. Math. Stat.

[9] John E. WaLSH, "On the power function of the sign test for slippage of means," Ann. Math. Stat., Vol. 17 (1946), pp. 358-362.

[10] JoHN E. WALSH, "On the asymptotic power efficiency of the sign test for slippage of means," Douglas Aircraft Co., Inc. Report.

[11] John E. Walsh, "Some comments on the efficiency of statistical tests," Human Biology, Vol. 21 (1949), pp. 205-217.

[12] JoHN E. WALSH, "Some significance tests for the median which are valid under very general conditions," Ann. Math. Stat., Vol. 20, (1949), pp. 64-81.

[13] John E. Walsi, "Some bounded significance level properties of the equal-tail sign test," Ann. Math. Stat., Vol 22 (1951), pp. 408-417.

\section{THE ADMISSIBILITY OF CERTAIN INVARIANT STATISTICAL TESTS INVOLVING A TRANSLATION PARAMETER}

\author{
By E. L. Lehmann ${ }^{r}$ and C. M. Stein \\ University of California, Berkeley, and University of Chicago
}

1. Introduction. The notion of invariance (or symmetry) has such strong intuitive appeal that many current statistical procedures have the invariance property and are in fact the best invariant procedures although they were pro-

Received 2/10/53.

${ }^{1}$ This work is supported in part by the Office of Naval Research. 
posed long before a general discussion of invariance was available. Hotelling [1], [2] and Pitman [3], [4] emphasized the invariant nature of certain tests and estimates. A general definition of the notion for the problem of testing hypotheses was given by Hunt and Stein who showed that in this case under severe restrictions on the group of transformations an optimum invariant test is most stringent or more generally minimax with respect to an invariant loss function (see [5]). This result has been extended to more general decision problems and more general groups by Peisakoff [6]. However, these results do not prove admissibility of the procedures in question unless the group of transformations is compact.

The problem of admissibility in the case of point estimation of a location parameter was treated in the normal case by Blyth [7] and by Hodges and I ehmann [8] and for a general class of location parameter-problems by Blackwell [9]. In the latter paper the surprising fact was brought to light that even in the location parameter problem the best invariant estimate may, under certain circumstances, be inadmissible.

In the present note we prove under conditions which are presumably unnecessarily restrictive the admissibility of the most powerful invariant test for testing one location parameter family against another. As an example, consider the problem in which $Z_{1}, \cdots, Z_{n}$ are normally distributed with unknown mean $\zeta$ and variance $\sigma^{2}$. If we wish to test $H: \zeta \leqq 0$ against the alternatives $K: \zeta>0$, it was already pointed out in ([5], p. 15) that Student's $t$-test is admissible for this problem. This result is quite elementary and rests on the fact that unbiasedness in this case implies that the probability of rejection equals the level of significance for all points $(\zeta, \sigma)$ with $\zeta=0$. However, this argument breaks down if we introduce an indifference zone and restrict our class of alternatives to $K^{\prime}: \zeta / \sigma \geqq \delta$ where $\delta$ is some specified positive number.

Consider now the general problem in which one observes a random point $(X, Y)$ where $X$ ranges over an arbitrary set, $Y$ over the real line. There are two hypotheses $H_{i}$ according to which the distribution of $(X, Y-\eta)$ is $F_{i}(=1,2)$ where $\eta$ is unspecified. The problem discussed above is an example of this, if we take $H_{i}$ to be $\zeta / \sigma=\delta_{i}, X=\sum Z_{i} / \sqrt{\sum Z_{j}^{2}}, Y=\log \sum Z_{j}^{2}$ and $\eta=\log \sigma$. As another example let $\left(Z_{1}-\eta, Z_{2}-\eta, \cdots, Z_{n}-\eta\right)$ have distribution $F_{i}$ under $H_{i}$. Then we can take for $X$ the set of differences $X=\left(Z_{1}-Z_{n}, \cdots, Z_{n-1}-Z_{n}\right)$ and for $Y$ the mean $\bar{Z}$ or the observation $Z_{n}$, or any of a number of other statistics.

2. The principal theorem. Let $x$ be a set (which for all practical purposes may be taken to be a Euclidean space), $a$ a $\sigma$-algebra of subsets of $x$ (say, the ordinary Borel sets if $X$ is Euclidean), $Q$ the real line, $B$ the set of all ordinary Borel subsets of $R, \lambda_{1}, \lambda_{2}$ probability measures on $Q$ and for each $x$, let $F_{1 x}, F_{2 x}$ be probability measures on $B$ such that for each $B \varepsilon B$, real $k$, and $i=1,2$ $\left\{x \mid F_{i x}(B) \leqq k\right\} \varepsilon a$. We suppose that the distribution of the random point $(X, Y)$ ranging over $X \times 9)$ is, for some real $\eta$, with $i=1$ or 2

$$
P_{i \eta}((X, Y) \varepsilon C)=\int_{C} d \lambda_{i}(x) \int d F_{i x}(y-\eta) .
$$


A test for the hypothesis $H_{1}$ that it is $P_{1 \eta}$ (with $\eta$ unspecified) is a function $\varphi$ on $x \times \mathbb{R}$ to $[0,1], Q B$ measurable. The test $\varphi$ is said to be better than $\varphi_{0}$ if for all $\eta$

$$
\begin{aligned}
E_{1 \eta} \varphi(X, Y) & \leqq E_{1 \eta} \varphi_{0}(X, Y) \\
E_{2 \eta} \varphi(X, Y) & \geqq E_{2 \eta} \varphi_{0}(X, Y),
\end{aligned}
$$

strictly better if (2) holds with strict inequality for some $\eta_{.} \varphi_{0}$ is admissible if there exists no $\varphi$ strictly better than $\varphi_{0}$.

TheOREM 1. If $E_{10}|Y|, E_{20}|Y|<\infty, 0<c<1$,

$$
\lambda_{1}\left\{x \mid \frac{d \lambda_{2}}{d\left(\lambda_{1}+\lambda_{2}\right)}(x)=c\right\}=0 \text {, }
$$

$\varphi_{0}$ is the test defined by

$$
\varphi_{0}(x, y)=\left\{\begin{array}{l}
1 \text { if } \frac{d \lambda_{2}}{d\left(\lambda_{1}+\lambda_{2}\right)}(x) \geqq c \\
0 \text { if } \frac{d \lambda_{2}}{d\left(\lambda_{1}+\lambda_{2}\right)}(x)<c,
\end{array}\right.
$$

and $\varphi$ is better than $\varphi_{0}$, then $\varphi-\varphi_{0}=0$ a.e. $\left(\lambda_{1}+\lambda_{2}\right) \mu$ where $\mu$ is ordinary Lebesgue measure on the real line.

Corollary. If in addition all $F_{i x}$ are absolutely continuous wrth respect to $\mu$, then $\varphi_{0}$ is admissible.

The corollary is an immediate consequence of Theorem 1.

Proof of Theorem 1. Putting $\lambda=\lambda_{1}+\lambda_{2}, f(x)=d \lambda_{2} / d\left(\lambda_{1}+\lambda_{2}\right)(x)$ we can rewrite the condition (2) that $\varphi$ be better than $\varphi_{0}$

$$
\begin{array}{r}
\int_{f(x)<c}(1-f(x)) d \lambda(x) \int\left(\varphi-\varphi_{0}\right)(x, y) d F_{1 x}(y-\eta) \\
-\int_{f(x) \geqq c}(1-f(x)) d \lambda(x) \int\left(\varphi_{0}-\varphi\right)(x, y) d F_{1 x}(y-\eta) \leqq 0 \\
-\int_{f(x)<c} f(x) d \lambda(x) \int\left(\varphi-\varphi_{0}\right)(x, y) d F_{2 x}(y-\eta) \\
\quad+\int_{f(x) \geqq c} f(x) d \lambda(x) \int\left(\varphi_{0}-\varphi\right)(x, y) d F_{2 x}(y-\eta) \leqq 0 .
\end{array}
$$

Multiplying (4) by $c$ and (5) by $1-c$ and adding we obtain

$$
\begin{aligned}
\int_{f(x)<c} c(1-f(x)) d \lambda(x) \int\left(\varphi-\varphi_{0}\right)(x, y) d F_{1 x}(y-\eta) \\
+\int_{f(x) \geqq c}(1-c) f(x) d \lambda(x) \int\left(\varphi_{0}-\varphi\right)(x, y) d F_{.2}(y-\eta) \\
\leqq \\
\quad \int_{f(x)<c}(1-c) f(x) d \lambda(x) \int\left(\varphi-\varphi_{0}\right)(x, y) d F_{2 x}(y-\eta) \\
+\int_{f(x) \geqq c} c(1-f(x)) d \lambda(x) \int\left(\varphi_{0}-\varphi\right)(x, y) d F_{1 x}(y-\eta) .
\end{aligned}
$$


In order to derive the conclusion of Theorem 1 from (6) we shall need the Lemma. If $x, a, a, B$ are as before, $\rho$ a probability measure on $a, h_{1}, h_{2} a$ measurable functions on $X$ to $[0,1]$ with $h_{1}-h_{2}>0$ a.e. $(\rho), \psi$ an aßB-measurable function on $X \times \&$ to $[0,1]$, and for each $x, H_{1 x}, H_{2 x}$ probability measures on $B$ such that

$$
\text { for each } B \varepsilon B \text { real } k \text { and } i=1,2,\left\{x \mid H_{i x}(B) \leqq k\right\} \varepsilon a
$$

$$
\begin{gathered}
\int h_{i}(x) d \rho(x) \int|y| d H_{i x}(y)<\infty . \\
\int h_{1}(x) d \rho(x) \int \psi(x, y) d H_{1 x}(y-\eta)
\end{gathered}
$$

$$
\leqq \int h_{2}(x) d \rho(x) \int \psi(x, y) d H_{2 x}(y-\eta) \quad \text { for all real } \eta
$$

then $\psi=0$ a.e. $(\rho \mu)$.

Proof of Lemma. We can rewrite (9)

(10) $\int h_{1}(x) d \rho(x) \int \psi(x, y+\eta) d H_{1 x}(y) \leqq \int h_{2}(x) d \rho(x) \int \psi(x, y+\eta) d H_{2 x}(y)$.

Now

$$
\begin{aligned}
\int_{-n}^{n} d \eta \int \psi(x, y+\eta) d H_{2 x}(y)-\int_{-n}^{n} \psi(x, \eta) d \eta \\
\quad=\int d H_{2 x}(y)\left[\int_{-n+y}^{n+y} \psi(x, \eta) d \eta-\int_{-n}^{n} \psi(x, \eta) d \eta\right] \\
\quad \leqq \int_{y \geqq 0} d H_{2 x}(y) \int_{n}^{n+y} \psi(x, \eta) d \eta+\int_{y \leqq 0} d H_{2 x}(y) \int_{-n+y}^{-n} \psi(x, \eta) d \eta \\
\quad \leqq \int|y| d H_{2 x}(y)
\end{aligned}
$$

and

$$
\begin{aligned}
\int_{-n}^{n} d \eta ; & \psi(x, y+\eta) d H_{1 x}(y)-\int_{-n}^{n} \psi(x, \eta) d \eta \\
& =\int d H_{1 x}(y)\left[\int_{-n+\nu}^{n+\nu} \psi(x, \eta) d \eta-\int_{n}^{n} \psi(x, \eta) d \eta\right] \\
& \geqq-\int_{y \geqq 0} d H_{1 x}(y) \int_{n}^{-n+\nu} \psi(x, \eta) d \eta-\int_{y \leqq 0} d H_{1 x}(y) \int_{n+\nu}^{n} \psi(x, \eta) d \eta \\
& \geqq-\int|y| d H_{1 x}(y) .
\end{aligned}
$$


Integrating (10) with respect to $\eta$ from $-n$ to $n$ and using the final forms of (11), (12) we obtain

$$
\begin{aligned}
& \int\left[h_{1}(x)-h_{2}(x)\right] d \rho(x) \int_{-n}^{n} \psi(x, \eta) d \eta \\
& \leqq \int h_{1}(x) d \rho(x) \int|y| d H_{1 x}(y)+\int h_{2}(x) d \rho(x) \int|y| d H_{2 x}(y) .
\end{aligned}
$$

Consequently,

$$
\int\left[h_{1}(x)-h_{2}(x)\right] d \rho(x) \int_{-\infty}^{\infty} \psi(x, \eta) d \eta<\infty
$$

and for every $\delta>0$ there exists $n$ such that

$$
\int\left[h_{1}(x)-h_{2}(x)\right] d \rho(x) \int_{|\eta| \geqq n} \psi(x, \eta) d \eta \leqq \delta .
$$

If instead of using the final forms of (11) and (12) for all $x$, we use them only in the range $h_{1}(x)-h_{2}(x)<\epsilon$ and use the next to final forms when $h_{1}(x)-h_{2}(x) \geqq \epsilon$ we obtain instead of (13).

$$
\begin{aligned}
& \int\left[h_{1}(x)-h_{2}(x)\right] d \rho(x) \int_{n}^{n} \psi(x, \eta) d \eta \\
& \leqq \int_{h_{1}(x)-h_{2}(x)<e} d \rho(x)\left[h_{1}(x) \int|y| d H_{1 x}(y)+h_{2}(x) \int|y| d H_{2 x}(y)\right] \\
& +\int_{h_{1}(x)-h_{2}(x) \geqq e} d \rho(x)\left[h _ { 1 } ( x ) \left\{\int_{y \geqq 0} d H_{1 x}(y) \int_{n}^{-n+y} \psi(x, \eta) d \eta\right.\right. \\
& \left.\quad+\int_{y \geqq 0} d H_{1 x}(y) \int_{n+y}^{n} \psi(x, \eta) d \eta\right\} \\
& \left.+h_{2}(x)\left\{\int_{y \geqq 0} d H_{2 x}(y) \int_{n}^{n+y} \psi(x, \eta) d \eta+\int_{\nu \leqq 0} d H_{2 x}(y) \int_{n+y}^{-n} \psi(x, \eta) d \eta\right\}\right] .
\end{aligned}
$$

The first term on the right-hand side can be made arbitrarily small by taking $\epsilon$ sufficiently small since $h_{1}(x)-h_{2}(x)>0$ a.e. $(\rho), 0 \leqq h_{i}(x) \leqq 1$ (using (8)). For, given $\epsilon>0$, the second half of the last term can be made arbitrarily small by choosing $n \geqq n(\epsilon)$ sufficiently large since by (15)

$$
\int_{h_{1}(x)-h_{2}(x) \geqq e} d \rho(x) \int_{|\eta| \geqq n} \psi(x, \eta) d \eta \leqq \delta / \epsilon .
$$

Also

$$
\begin{aligned}
& \int_{h_{1}(x)-h_{2}(x) \geqq e} d \rho(x) h_{1}(x) \int_{n / 2 \leq y} d H_{1 x}(y) \int_{-n}^{-n+y}-\psi(x, \eta) d \eta \\
& \leqq \int_{h_{1}(x)-h_{2}(x) \geqq e} d \rho(x) h_{1}(x) \int_{n / 2 \leqq \nu} y d H_{1 x}(y) .
\end{aligned}
$$


Again, for fixed $\epsilon$ this can be made arbitrarily small by choosing $n \geqq n(\epsilon)$ sufficiently large. Finally

$$
\begin{aligned}
\int_{h_{1}(x)-h_{2}(x) \geqq e} d \rho(x) h_{1}(x) \int_{0}^{n / 2} d H_{1 x}(y) & \int_{-n}^{-n+y} \psi(x, \eta) d \eta \\
& \leqq \int_{h_{1}(x)-h_{2}(x) \geqq e} d \rho(x) h_{1}(x) \int_{-\infty}^{-n / 2} \psi(x, \eta) d \eta,
\end{aligned}
$$

which is disposed of in the same way as the second half of the last term. The remaining integral with $y \leqq 0$ is analogous. Then, since the right-hand side of (16) is arbitrarily small for sufficiently large $n, \psi=0$ a.e. $\left(\lambda_{1}+\lambda_{2}\right) \mu$. This completes the prof of the Lemma.

To apply the Lemma to (6) we make the following identifications:

(i) If $f(x)<c$,

$$
\begin{array}{rlrl}
h_{1}(x) & =c(1-f(x)) & h_{2}(x)=(1-c) f(x) \\
H_{1 x} & =F_{1 x} & H_{2 x}=F_{2 x} . \\
\psi & =\varphi-\varphi_{0} & &
\end{array}
$$

(ii) If $f(x) \geqq c$

$$
\begin{array}{rlrl}
h_{1}(x) & =(1-c) f(x) & h_{2}(x) & =c(1-f(x)) \\
H_{1 x} & =F_{2 x} . & H_{2 x}=F_{1 x} . \\
\psi & =\varphi_{0}-\varphi & &
\end{array}
$$

In any case $\rho=\lambda / 2$. The reader will readily verify that (7), (8), (9) are satisfied so that the theorem follows.

A moment's reflection shows that the origin of $Q$ for given $x$ is arbitrary so that the hypotheses $E_{\text {io }}|Y|<\infty$ could be replaced by: There exists an $Q$-measurable real-valued function $\tau$ on $X$ such that $E_{i 0}|Y-\tau(X)|<\infty$.

It is seen that the admissibility of the noncentral $t$-test for testing $\zeta / \sigma=\delta_{0}$ against $\zeta / \sigma=\delta$, (central in case $\delta_{0}=0$ ) follows immediately from the theorem since

$$
E\left|\log \sum Z_{1}^{2}\right|<\infty
$$

and $P\left(\sum Z_{i}=c \sqrt{\left.\sum Z_{i}^{2}\right)}=0\right.$.

Another example is that of testing for the same random variables $\sigma=\sigma_{0}$ against $\sigma=\sigma_{1}$. Here we may take $X=\sum\left(Z_{i}-\bar{Z}\right)^{2}$. and $Y=\sum Z_{i}$. Actually in this case the result can be proved quite easily by other means. Instead of taking for $\zeta$ the usual least favorable sequence of a priori distributions which in the limit is invariant, we may, if $\sigma_{0}<\sigma_{1}$ take in $H$ the a priori distribution $P(\zeta=a)=1$ where $a$ is any constant, and in $K$ a normal distribution with mean $a$ and variance $n\left(1 / \sigma_{1}^{2}-1 / \sigma_{0}^{2}\right)$. The Bayes solution is seen to be the $F$-test which is therefore admissible. (For details see [10]). 
We can also consider the general linear hypothesis with no unknown means as nuisance parameters. For brevity we use the terminology of [5]. In the canonical form we have $U_{1} \cdots U_{m}, V_{1} \cdots V_{n}$ independently normally distributed with $E U_{i}=v_{i}, E V_{j}=0, E\left(U_{i}-v_{i}\right)^{2}=E V_{j}^{2}=\sigma^{2}$ where $\sigma^{2}, \psi_{i}$ are unknown and we want to test the hypothesis that all $v_{i}=0$ say, against $\sum v_{i}^{2} \geqq \gamma \sigma^{2}$. A sufficient statistic is $\left(U_{1} \cdots U_{m}, \sum V_{j}^{2}\right)$. The problem is invariant under rotation of the vector $U_{1}, \cdots, U_{m}$ and multiplication of all $U_{i}, V_{j}$ by the same constant $c$. Since the rotation group $G_{0}$ possesses a finite invariant measure, any test invariant under $G_{0}$ and admissible among all tests invariant under $G_{0}$ is admissible. Thus, in proving the usual $F$-test admissible we may restrict our attention to tests depending only on $\left(\sum U_{i}^{2}, \sum V_{j}^{2}\right)$. Under multiplication by $c$ this goes into $\left(c^{2} \sum U_{i}^{2}, c^{2} \sum V_{j}^{2}\right)$. Taking $X=\sum U_{i}^{2} / \sum V_{j}^{2}$ and $Y=\log \sum V_{j}^{2}$, applying Theorem 1 and the optimum property of the $F$-test among all.those based only on $\sum U_{i}^{2}$ / $\sum V_{j}^{2}$, we obtain the admissibility of the usual test: Reject $H_{0}$ if $\sum U_{i}^{2} \geqq k \sum V_{j}^{2}$. The same argument applies to the problem of testing $H_{0}: \sum v_{i}^{2} \leqq \gamma_{1} \sigma^{2}$ against $H_{1}: \sum v_{i}^{2} \geqq \gamma_{2} \sigma^{2}$ with $\gamma_{2}>\gamma_{1}$.

\section{REFERENCES}

[1] H. Hotelling, "Analysis of a complex of statistical variables into principal components," J. Educational Psychology, Vol. 24 (1933), pp. 417-441 and pp. 498-520.

[2] H. Hotelling, "Relations between two sets of variates," Biometrika, Vol. 28 (1936), pp. 321-377.

[3] E. J. G. Prtman, "The estimation of the location and scale parameters of a continuous population of any given form," Biometrika, Vol. 30 (1938), pp. 391-421.

[4] E. J. G. Pitman, "Tests of hypotheses concerning location and scale parameters, Biometrika, Vol. 31 (1939), pp. 200-215.

[5] E. L. Lehmann, "Some principles of the theory of testing hypotheses," Ann. Math. Stat., Vol. 21 (1950), pp. 1-26.

[6] M. Prisaroff, Unpublished Ph.D. thesis, Princeton University, 1950.

[7] C. Blrth, "On minimax statistical decision procedures and their admissibility," Ann. Math. Stat., Vol. 22 (1951), pp. 22-42.

[8] J. L. Hodges, JR. AND E. L. LehmanN, "Some applications of the Cramer-Rao inequality," Proceedings of the Second Berkeley Symposium on Mathematical Statistics and Probability, University of California Press, 1951.

[9] D. Blackwell, "On the translation parameter problem for discrete variables," Ann. Math. Stat., Vol. 22 (1951), pp. 393-399.

[10] E. L. Lehmann and C. Strein, "Most powerful tests of composite hypotheses, I. Normal distributions," Ann. Math. Stat., Vol. 19 (1948), pp. 495-516. 